\title{
Chaos: Challenges From and To Socio-Spatial Form and Policy
}

\author{
DIMITRIOS S. DENDRINOS \\ Urban and Transportation Dynamics Laboratory, 107 Marvin Hall, The University of Kansas, Lawrence, KS 66045-2250, USA
}

(Received 9 October 1996)

\begin{abstract}
A brief assessment is given of the major accomplishments made through the mathematics of chaos to the understanding of socio-spatial dynamics to date. Certain shortfalls are also presented, mostly associated with model testing and falsifiability which transcend socio-spatial dynamics. Beyond such shortcomings, lie an array of challenges for chaotic dynamics involving specifically socio-spatial form and policy. A few directions on meeting these challenges are suggested including the case of limited chaos.
\end{abstract}

Keywords: Chaos, Socio-spatial dynamics, Complexity, Entropy, Limited chaos

\section{INTRODUCTION}

This is the inaugural issue of a journal set up to carry out a formidable and unique experiment: to search into the possibility of integrating the sociospatial and natural sciences through the (universal?) method of discrete chaotic dynamics or, more broadly, nonlinear dynamics. No doubt, such an attempt will be met by considerable challenges. By and large, the first challenge will be to demonstrate the validity of such a method for socio-spatial analysis beyond mere speculative theoretical hypotheses, by no means an easy task.

Socio-spatial dynamics lack a BelousovZhabotinskii chemical reaction, which so clearly demonstrates the emergence of pattern out of well understood nonlinear interactive dynamics off equilibrium states with such an impressive and eloquent manner. No matter how much socio-spatial analysts yearn for and seek equivalently demonstrative examples (by looking for instance into stock market time series or urban traffic flow patterns), socio-spatial evidence carrying such convincing power may never come about. Even if one argues that socio-spatial analysts are looking in all the wrong places, there can be very little doubt that if any good places or times to look for such patterns exist, there must be very rare if any at all: social reality simply is not that neat. This is the null hypothesis.

Those of us in the social sciences in general, and the human spatial sciences in specific, who choose to use nonlinear dynamics in modeling and interpreting socio-spatial events in a manner which draws from the power and (albeit nonsocial) beauty of mathematical chaos have done 
so thus far merely out of eclectic preferences, and on obviously tenuous grounds. And we will be the first to admit it. In our speculative journeys, we search for qualitative properties and rigorous mathematical statements about socio-spatial dynamics utilizing certain key elements of chaos. It is of interest to note that all of us who approach socio-spatial dynamics from this perspective come from different angles (initial conditions). We do so only because something quite compelling attracts us to it. A faint hope and possibly weak expectation characterizes our quest, that somehow we are to acquire innovative insight from such an endeavor.

But why are we so much drawn by mathematical chaos? Aesthetic appeal and fashion arguments aside, it is because of its promise: we are betting that when and if such insight does ever reveal itself and is appreciated by a wide enough audience, it will have a powerful and profound impact upon social action. It will compare only to that brought about by Malthus, the end of whom this construct indeed foretells. And social action is the ultimate motivation of all social scientists, transcending all social theories, no matter how well these theories fit the real world. After all, real world phase space and model world phase space are not, and they may never be topologically equivalent.

\section{SOCIO-SPATIAL COMPLEXITY}

For long, social sciences have acknowledged the obvious, namely that the key ingredient in the soup of social systems, as well as in social action (at both the individual and collective levels) is complexity. Far beyond adding flavor to social systems and actions, complexity is contained in them for good reason: it offers them a high degree of freedom, and it is as if these systems and actions have an inherent and inalienable right to complexity, a multidimensional concept.

To the immense micro- and macro-human heterogeneity, if one adds the vast spatial hetero- geneity of our planet in its micro- and macrogeographies, and superimposes all this to the startling differences in micro- and macro-socioglobal time periods - in all what we like to call "social spatio-temporal heterogeneity" - then one begins to appreciate our predicament and quest in finding simple patterns out of the complexity of human behavior. Incidentally, this spatio-temporal heterogeneity is a strong enough reason to simulate socio-spatial dynamics in discrete space and time, hence the title of this journal.

We are forced to seek any help we can get (let alone inspiration) from all sources, including the natural sciences and mathematics. Mathematical modeling of complex systems seeks to find and isolate simple patterns of behavior to at least formulate some hypotheses at some aggregative (coarse) scale, hypotheses which we find of interest for various reasons and within certain contexts.

Our interest in mathematical chaos can be well founded by drawing on certain very articulate arguments made in an interesting and recent book by Cohen and Stewart (1994). There, the authors try to derive some general principles on how to link simplicity of laws governing the behavior of physical systems to their underlying complexity. They do so because they wish to go beyond natural sciences and its reductionist methods, and derive a general theory of complexity which could encompass the social sciences as well. Simplicity in aggregate behavior of certain systems can be and has been detected, the authors argue, while the disaggregative (fine breakdown into) parts of these systems are involved into a complex interplay oftentimes far beyond our full understanding, let alone our capabilities to model.

Certain qualitative properties of chaotic dynamics, sensitivity to initial conditions and the presence of chaotic attractors in particular, seem to stimulate suggestive linkages between the simple upper level laws of nature and the complex lower level interplay of the numerous parts of a system under investigation. To Cohen and Stewart these linkages between complexity and simplicity are of 
special interest because they seem to also suggest an alternative (nonreductionist) way for approaching the study of these systems. Nonreductionism is of interest to socio-spatial analysts as they encounter considerable obstacles in being pure reductionist of any note.

Cohen and Stewart devised two new concepts, "simplexity" and "complicity" to show how, depending on a context, two types of patterns can possibly emerge in the study of complex systems. The first pattern is drawn from the legacy of chaos which highlights the extreme dependency to initial conditions. Simplexity emerges when an inherently (at a lower level of analysis) complex system, comprised of a vast number of almost identical parts (rules), has simple (upper level) laws (one outcome) which describes it well given our context of choice. The perennial example of Newton's law of gravitation is cited, given how it can (accurately to an acceptable approximation) describe any planet's orbit, although a planet is an aggregate of a very large number of (almost identical in their position in space when viewed from a long enough distance) individual particles. On the other hand, complicity emerges when a set of many vastly different parts (rules) can generate a simple aggregate outcome. This case highlights the presence of specific strange attractors in phase space of dynamical systems under a chaotic regime.

This approach to complexity is clearly one of the legacies of mathematical chaos. Cohen and Stewart refer to complicity as chaos "collapsed" and they speculate that the still elusive Theory of Everything in physics will contain such a complicity pattern. They go even further and attribute this mode of thought to the manner in which the human mind (or consciousness) really works. Well, a social scientist might not be so sure about the last claim, but few will doubt the fruitfulness of a search for a nonreductionist complicity type model of human societies.

All the efforts recorded so far by socio-spatial analysts, see the latest work contained in a Special Issue on "Nonlinear Dynamics in Urban and
Transportation Analysis" in the journal Chaos, Solitons and Fractals (1994), employing concepts and ideas from chaotic dynamics, point to this nonreductionist premise as their pure (and possibly only) justification. Be that inter- or intraurban population dynamics, dynamical central place theory, dynamics of urban form and structure, inter-regional linkages through trade dynamics, technological spatio-temporal diffusion, or any other socio-spatial topic attracting current attention in the literature, all share a common starting point: the suggested model form does not emerge out of extensive experimentation or through analyzing vast data sets, but rather out of theoretical speculation.

\section{THE CHALLENGES}

There is still no empirical evidence to make the transition to a reductionist mode, while dealing with social dynamics under any social science perspective, no matter the numerous claims to the contrary from many a source. In that sense, we are forced into seeking rigorous nonreductionist theories. No matter their theoretical pedigree, some theoretical propositions enjoy a wider recognition simply because social scientists have liberal degrees of tolerance for approximations their models and outputs carry with reality, a tolerance which in any case is quite higher than that required for modeling in the natural sciences.

To the extent that the social mathematical chaos literature is concerned, all available statistical evidence presented to date is at least inconclusive, see also the book by Kiel and Elliott (1995), where some evidence from economics is assessed with similar conclusions. What may seem like patterns which, if allowed to go on for a long enough time period, may demonstrate chaotic behavior and fractal properties can also be viewed as mere oscillations, indistinguishable from various types of noise. It is so in stock market data and in traffic flow data, two social time series that are 
available in relatively frequent counts to even start asking testable questions.

In reality there is $\chi \alpha \alpha o \sigma$, but it does not possess the properties (or the oddities as some might say) of mathematical chaos. But this difference may not be as damaging as it may sound at first and, to the contrary, it might even be quite helpful to better understand both real chaos and reality. For instance, what is (in reality) determinism, randomness and uncertainty may be better understood by looking at the deterministic structure of simple chaos bearing mathematical models.

Efforts to unpeel "layers of determinism" led Kellert (1993) into envisioning "bounds to predictability" for complex systems even simply stated ones, in presence of apparent order under the chaos demon, and thus rendering our understanding of these systems quite limited let alone discrediting any efforts towards prediction. Chaotic regimes point out dramatically how errors in estimating the state variables might be more due to the errors in the estimation of parameters (primarily due to levels of approximation involved in such parameter estimates aside from their mis-specification), so much so that they might compromise errors in the state variable(s) due to slight shift in initial conditions (or their approximations). And all this, in addition to model mis-specification difficulties, which are rendered almost impossible under chaotic conditions. In effect, chaos points to the flaws of the very method of estimation.

\section{PROSPECTS}

This journal's tall order, to attempt an integration through discrete nonlinear dynamics of the natural and socio-spatial sciences by disproving the null hypothesis, must however proceed because of the high stakes involved, even if the prospects as of now for its eventual success may be judged to be limited. The reasons are indeed quite obvious, and of potential interest to natural scientists as well: if Physics can produce a Theory of Everything this Grand Theory must link all components of this Universe at all its scales, from the nanoscale to the cosmological scale through the mesoscale of human societies; no matter how informative such a Theory might turn out to be it must address our scale too.

And if a Theory of Evolution is such a general theory as its advocates proclaim, then such a theory must link all scales too, and in particular all these mesoscales neighboring our own scale at the level of human societies.

So far, and beyond these grand themes, the general method of nonlinear interactive dynamics has failed to address subjects of immediate and practical interest to those who directly impact socio-spatial form, structure and policy or action. The form and architecture of buildings, settlements, cities, nations; the structure (economic, social, political or any other one) of individuals, communities or any other social aggregates; the concrete social actions or policies by individuals and governments, all of the above have remained aloof from the plows of nonlinear interactive dynamics and chaos. The challenge of nonlinear dynamics is to demonstrate its relevance to concrete policy, management and design.

Socio-spatial dynamics have remained so far too abstract; efforts to produce certain universal rules have neglected (some even would argue that nonlinear dynamics are incapable of) addressing specific subjects of social systems because of its generality, its complicity. Maybe, like the expected Theory of Everything, it might illuminate us on nothing. But this criticism could be leveled against all mathematical modeling of socio -spatial systems. Be that as it may, socio-spatial dynamics must first demonstrate that they are not more guilty of such neglect than at least the average mathematical model of social systems. And after having done so then they can march ahead.

\section{CERTAIN SUGGESTIONS}

Numerous lines of promising research are currently underway, some presented in the 1994 issue 
of Chaos, Solitons and Fractals, as already mentioned. Here, I will confine myself to only two possible outlets for fruitful extensions: the subject of entropy in socio-spatial systems, and the subject of limited chaos in a largely interconnected network of spatio-temporally interacting stocks.

How is entropy, of the information type a la Shannon, behaving in socio-spatial systems and what principles of extremum are implied in such governing potentials (if any) is a subject of some interest to social analysis. For one, it supplies a possible linkage between the natural and social sciences, as indicated for example by the two Editors of this journal, Sonis and Gontar (1992). One would like to see, however, what is the exact interpretation of these principles and what do they imply for social action under specific contexts.

The second subject is that of limited chaos, a topic which has not attracted much attention among either mathematicians or natural scientists, let alone social scientists, and first identified by Dendrinos and Sonis (1990) in the case of a general discrete nonlinear relative dynamics map containing interactions among one stock' shares

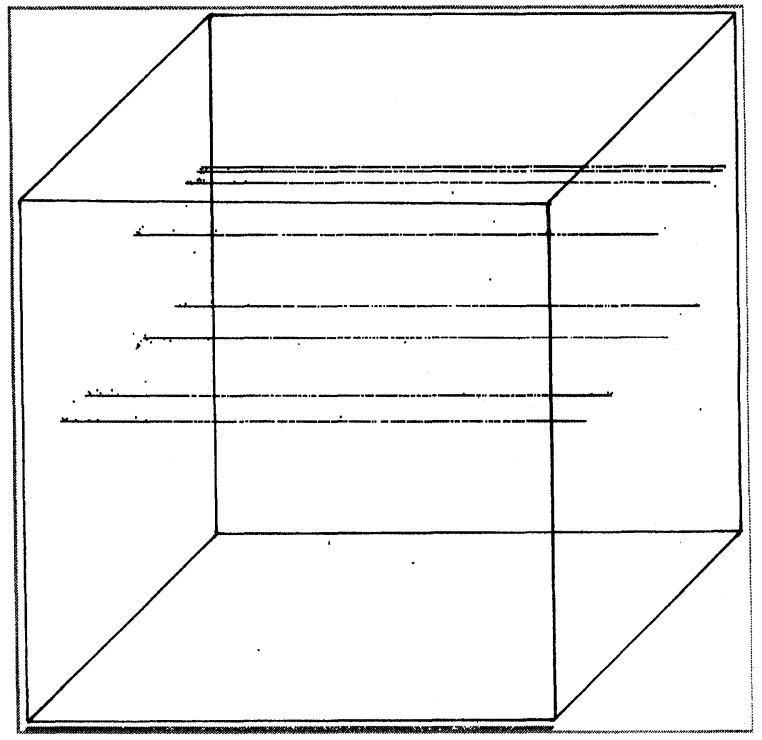

FIGURE 1 Rod-type dynamics of limited chaos; 10,000 iterations. See text for parameter specifications. distributed over three locations. More broadly, it is possible to show that in a rather extensively interactive nonlinear dynamical system, some state variables might experience chaotic behavior while others are in relatively calm dynamics. Some examples of this phenomenon are shown in Figs. 1, 2: in Fig. 1 the case of a "rod" type, and in Fig. 2 the case of a "sheet" type dynamics are given. Both cases correspond to limited chaos plotted on the unit cube, and are based on the three stocks and two locations version of the map, see Dendrinos and Sonis (1990):

$$
x_{i}(t+1)=F_{i}(t) /\left[1+F_{i}(t)\right], \quad i=1,2,3,
$$

where

$$
\begin{aligned}
& \ln F_{i}(t)=\ln A_{i}+\sum_{i}\left[a_{i j} \ln x_{j}(t)+b_{i j} \ln \left(1-x_{j}(t)\right],\right. \\
& \quad i, j=1,2,3, \\
& \quad 0<x_{i}(t)<1, \quad F_{i}(t)>0, A_{i}>0, \\
& \quad-\infty<\left(a_{i j}, b_{i j}\right)<+\infty .
\end{aligned}
$$

In this map, $F_{i}(t)$ depicts the locational comparative advantages (a Darwinian notion) of stock $x_{i}$

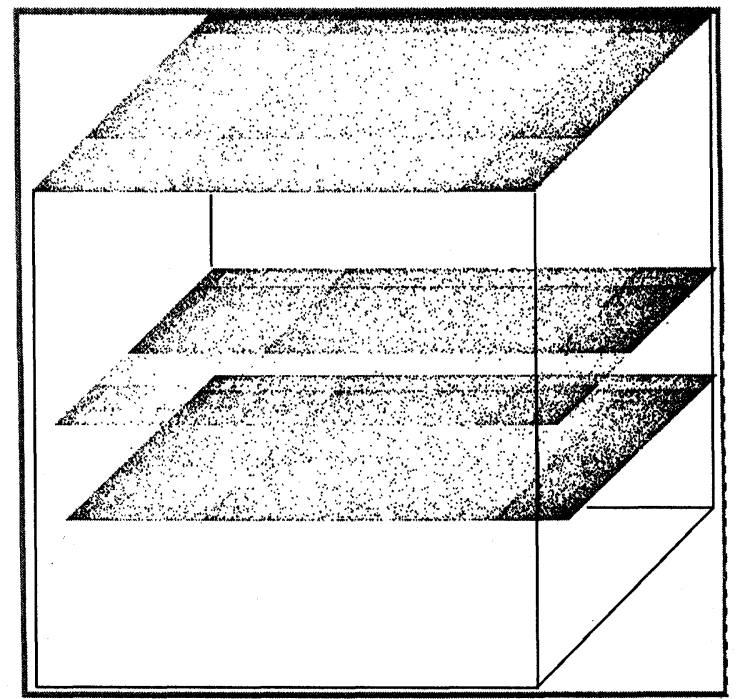

FIGURE 2 Sheet-type dynamics of limited chaos; 10,000 iterations. See text for parameter specifications. 
at a designated location (one of two competing for the three stocks locations) at time $t$. Parameters a and $\mathbf{b}$ designate elasticities of interaction (composites of the prevailing interaction forces, a physical notion) among stocks and between locations (due to both size and location). The parameters $\mathbf{A}$ identify environmental constants (representing different socio-spatial universes).

Under the parameter specifications:

$$
\begin{aligned}
{[\mathbf{a}] } & =\left[\begin{array}{ccc}
-2 & 2 & 0 \\
0 & -2 & 0 \\
0 & 0 & -2
\end{array}\right], \\
{[\mathbf{b}] } & =\left[\begin{array}{ccc}
-1 & 0 & 0 \\
0 & -1 & 0 \\
0 & -1 & -2
\end{array}\right], \\
\mathbf{A} & =\left(10^{-4}, 10^{-4}, 10^{-3}\right), \quad \mathbf{x}(0)=(0.3,0.1,0.2),
\end{aligned}
$$

one obtains the "rod" type dynamics of Fig. 1, identifying a two-dimensional (for stocks $x_{2}$ and $x_{3}$ ) set of points where trajectories are attracted (stable periodic motion), and nonattractor bound chaotic behavior for the $x_{1}$ stock.

In Fig. 2, and under the specifications:

$$
\begin{aligned}
{[\mathbf{a}] } & =\left[\begin{array}{ccc}
-2 & 0.2 & 0 \\
0 & -2 & 0 \\
0 & 0 & -1
\end{array}\right], \\
{[\mathbf{b}] } & =\left[\begin{array}{ccc}
-1 & 0 & 0 \\
0 & -1 & 0 \\
0 & -1 & -1
\end{array}\right], \\
\mathbf{A} & =\left(10^{-4}, 10^{-4}, 10^{-3}\right), \quad \mathbf{x}(0)=(0.3,0.1,0.2),
\end{aligned}
$$

a "sheet" type dynamics takes place, whereby a small set of points exist in one dimension $\left(x_{3}\right)$ towards which trajectories are attracted (stable periodic movement), while undergoing nonattractor bound chaotic behavior along the other two dimensions $\left(x_{1}, x_{2}\right)$.

Thus, one or two stocks exhibit some periodic motion, referred to as "calm" dynamics, while the other (two or one stock respectively) are undergoing isolated or limited turbulent dynamics. These examples demonstrate another dimension of the universality of the map in depicting a variety of qualitatively different dynamics, a subject that could be of interest to the contributors of this journal.

Another topic, partly related to the cases discussed above and central to the objective of this journal, is the manner in which the generality of this map can be employed in the natural sciences as well. In Fig. 3, an example is given of how this three-stock two-location statement of the general stock-location interaction map can, under chaotic regimes without any recognizable attractors in phase space, generate inside the unit cube distributions reminiscent of the manner in which certain cosmological models envision visible matter in the universe to be distributed over

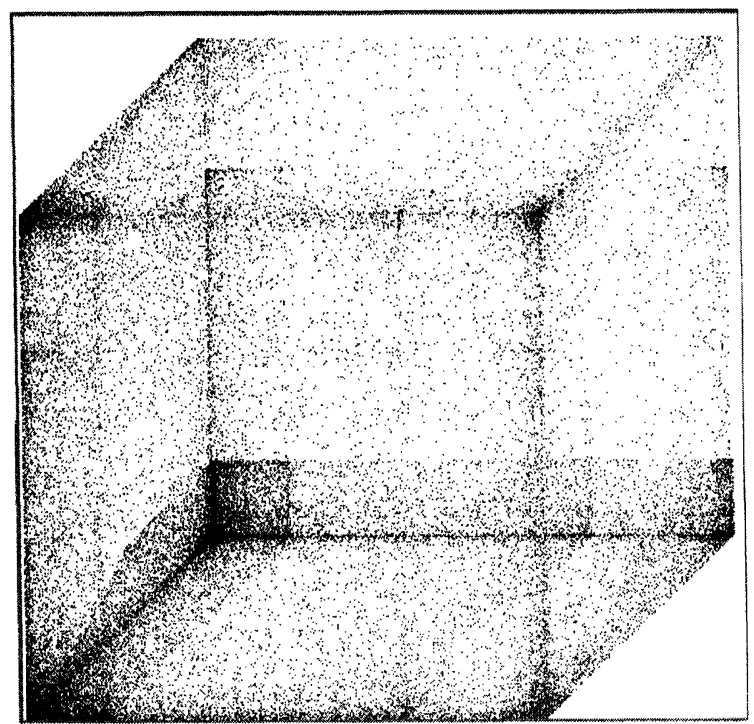

FIGURE 3 Chaotic dynamics in the unit cube in absence of attractors; 25,000 iterations. See text for parameter specifications. 
three-dimensional space. Figure 3 is generated under the specifications:

$$
\begin{aligned}
{[\mathbf{a}] } & =\left[\begin{array}{ccc}
-1.5 & -1 & 0 \\
0 & -2 & 0 \\
0 & 0 & 0
\end{array}\right], \\
{[\mathbf{b}] } & =\left[\begin{array}{ccc}
-1.5 & 0 & 0 \\
0 & -2 & 0 \\
0 & 0 & -2
\end{array}\right], \\
\mathbf{A} & =\left(10^{-4}, 10^{-4}, 10^{-6}\right), \quad \mathbf{x}(0)=(0.3,0.1,0.2) .
\end{aligned}
$$

\section{CONCLUSIONS}

Mathematical modeling in the social sciences has been in place for about a century now. Beyond acting as an intellectual pilot, mathematical modeling must move ahead and show its capacity to become the heavy duty container of commodities. If this is not achieved in short order, doubts regarding the sustainability of such an intellectual activity will increase. These doubts will further erode the credibility base of social sciences, and certainly it will impact upon its competitiveness in being supported by the collective resources of human societies. A failure of this magnitude will enforce some social scientists fears that social theorizing is (and should be regarded accordingly as) much more of an individual activity rather than a collective (public) good.

Nonlinear interactive dynamics modeling in the socio-spatial sciences presents an excellent forum for such a resolution to be abjudicated. The task ahead is to show beyond a reasonable doubt that socio-spatial systems do indeed contain chaos rather than $\chi \alpha$ ó $\sigma$.

\section{Acknowledgement}

The author wishes to thank his research assistant Terry Zhao, who in the Fall of 1992 produced the computer graphics shown in this paper.

\section{References}

Chaos, Solitons and Fractals (1994). Special Issue on nonlinear dynamics in urban and transportation analysis, 4(4).

J. Cohen and I. Stewart (1994). The Collapse of Chaos, Viking, New York.

D.S. Dendrinos and M. Sonis (1990). Chaos and Socio-Spatial Dynamics, Springer-Verlag, New York.

S.H. Kellert (1993). In the Wake of Chaos, University of Chicago Press, Chicago.

L.D. Kiel and E. Elliott (1995). Chaos Theory in the Social Sciences; Foundations and Applications, Michigan University Press, Ann Arbor.

M. Sonis and V. Gontar (1992). Entropy principle of extremality, Socio-Spatial Dynamics, 3(2), 61-73. 


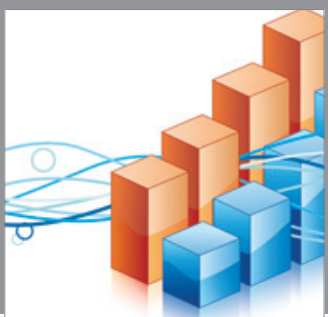

Advances in

Operations Research

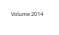

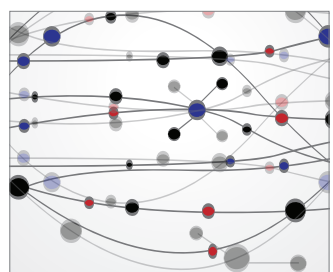

\section{The Scientific} World Journal
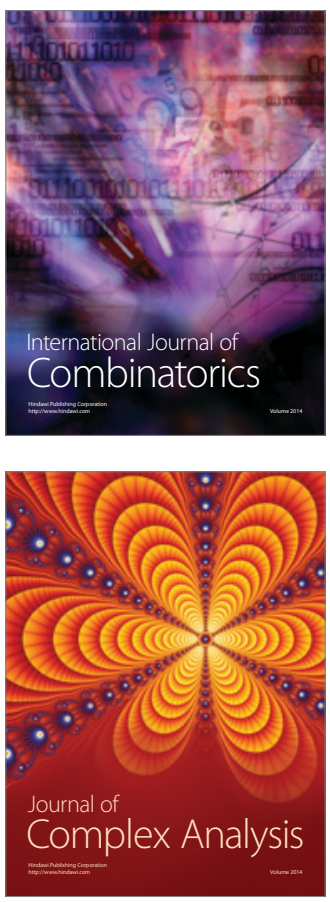

International Journal of

Mathematics and

Mathematical

Sciences
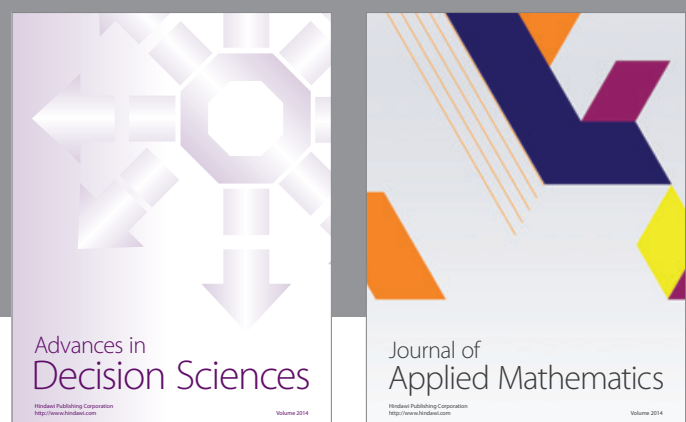

Journal of

Applied Mathematics
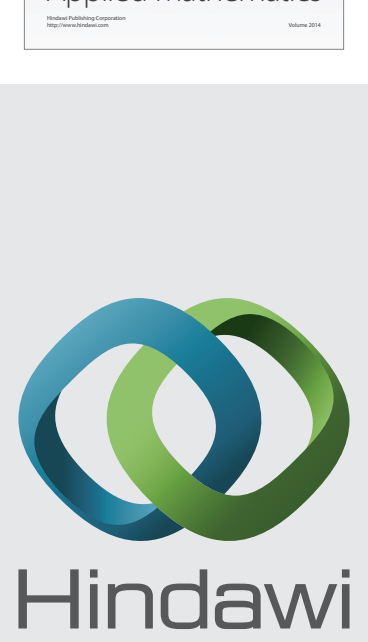

Submit your manuscripts at http://www.hindawi.com
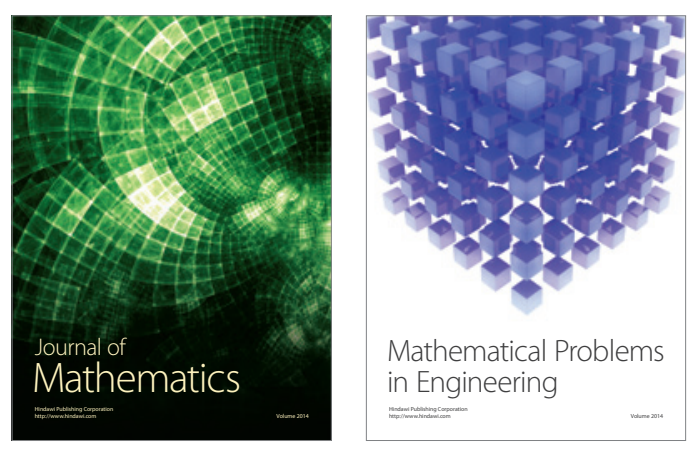

Mathematical Problems in Engineering
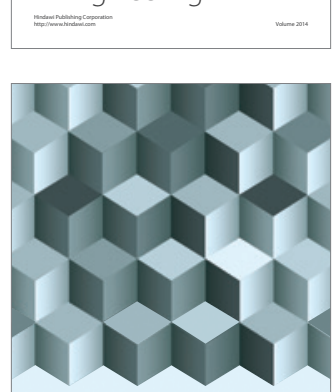

Journal of

Function Spaces
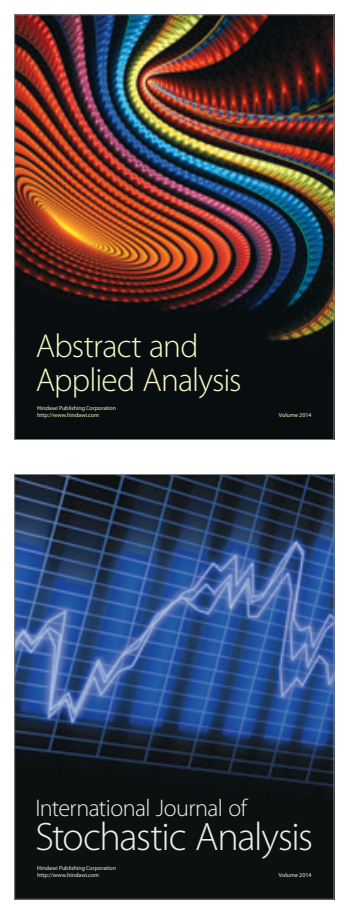

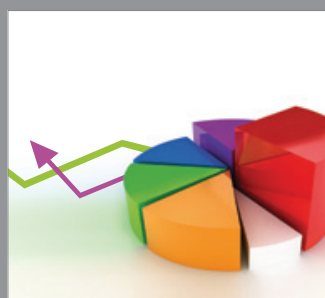

ournal of

Probability and Statistics

Promensencen
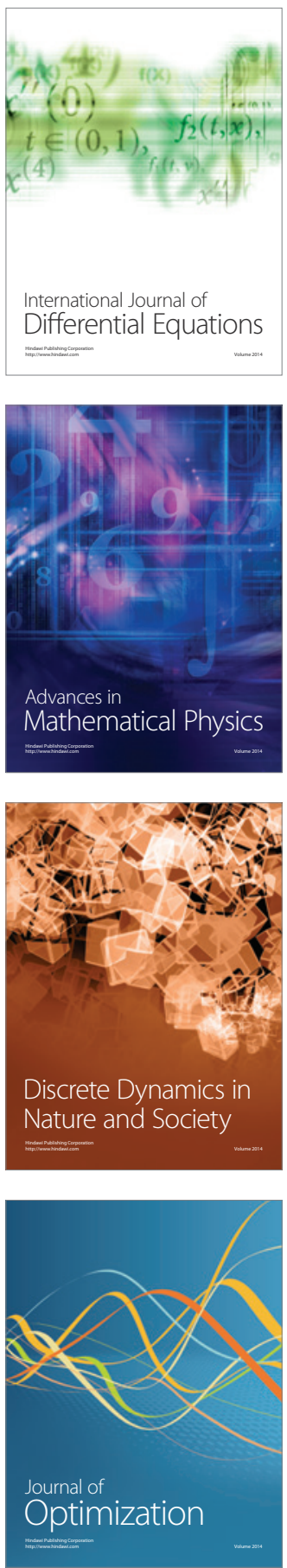